\title{
EFECTO DE LA ESCARIFICACIÓN QUÍMICA EN LA CALIDAD DE SEMILLA DE Centrosema SPP. DURANTE EL ALMACENAMIENTO ${ }^{1}$
}

\author{
Edgar A. Búrbano ${ }^{2}$
}

\section{COMPENDIO}

Centrosema spp. es uno de los géneros más importantes dentro de las accesiones de leguminosas forrajeras en el área de impacto de suelos ácidos e infértiles del trópico. En estos materiales se presenta un alto porcentaje de semillas duras, que aún al proporcionar las condiciones óptimas para que germine no lo hace naturalmente. Con tal fin se seleccionaron tres especies: $C$. acutifolium, C. macrocarpum y C. brasilianum, para evaluar el efecto de la escarificación química en la germinación y emergencia en el campo y 19 meses de almacenamiento, bajo condiciones controladas $\left(18^{\circ} \mathrm{C}\right.$ y $50 \%$ H.R.) y al ambiente; comparar el comportamiento de la semilla sin y con escarificación y analizar la interacción de especies por tratamiento. Los resultados mostraron que $C$. acutifolium fue la especie que perdió más rápidamente la germinación en los diferentes tratamientos (40\% a los cinco meses de almacenada). Las otras dos especies presentan mayor dureza, lo cual permite que la caída de la germinación no sea tan acentuada (10\% máximo de pérdida). Los resultados de emergencia en el campo fueron diferentes a los valores de germinación en el laboratorio (aproximadamente un 50\%). Los resultados permiten concluir que semillas almacenadas bajo los dos tipos de almacenamiento para forrajeras como Centrosema no superan su dureza significativamente. Las condiciones controladas favorecen la calidad fisiológica (germinación), manteniendo la dureza como en el caso de C. macrocarpum. De igual forma no se debe guardar semilla escarificada bajo condiciones no controladas, como en el caso de C. acutifolium que al perder rápidamente su dureza no presenta mecanismos de defensa para evitar el deterioro durante el almacenamiento. Si se pretende escarificar unos días antes de la siembra, se necesita tener condiciones de almacenamiento adecuadas.

Palabras clave adicionales: Leguminosa, forrajes, C. macrocarpum; C. acutifolium.

\section{INTRODUCCIÓN}

La producción de semillas de leguminosas forrajeras tropicales es muy variable, dependiendo de la región de producción y, como consecuencia, sus rendimientos no son muy consistentes.

El género Centrosema es de mucho interés para el Programa de Pastos Tropicales del CIAT. A la fecha se ha liberado el Centrosema acutifolium CIAT No. 5277 cultivar Vichada, el cual presenta características favorables de pastoreo en la zona de la altillanura de Colombia, caracterizada por suelos de baja fertilidad. Dependiendo de las características climáticas, edáficas, de manejo y económicas, se puede desarrollar un programa de producción de semillas que garantice una mínima producción. La actitud corriente entre la mayoría de los ganaderos es la de adquirir semillas de un cultivar

\begin{abstract}
${ }^{3}$
One of the most important forage legumes for acid and unfertile tropical soils is Centrosema spp. This genus is characterized by extremely hard seeds. Even with optimum conditions, because of its hard seeds, much does not germinate naturally. With this in mind, three species were selected, C. acutifolium, C. macrocarpum and $C$. brasilianum to evaluate the effect of chemical scarification on germination and emergence. Also, germination and emergence for these three entries were evaluated after having been stored for nineteen months at $18^{\circ} \mathrm{C}$ and $50 \%$ relative humidity. Seed counts with and without scarification and the associated interaction with the defined treatments. The results indicated germination was lowered more with Centrosema acutifolium than the others when the described treatments were assessed (germination lowered by $40 \%$ after five months of storage). The other two species have harder seed which reflects the limited loss In germination observed (maximum of $10 \%)$. The emergence results from field trials were substantially different from germination data obtained in the laboratory (approximately 50\%). These results indicate that seed stored under the two treatments for forage species such as Centrosema spp. Did not improve hardening significantly. The controlled conditions favored physiological qualities (germination) while maintaining hardness which was the case for $C$. macrocarpum. AWN scarified seed should not be stored under non controlled conditions as was noted for $C$. acutifolium which lost hardness rapidly and lacks defense mechanisms to avoid deterioration while stored. If scarification is employed a few days prior to seeding, adequate storage conditions should be employed.
\end{abstract}

en particular, al precio del mercado, sin reparar el valor potencial o valor real de la semilla, su pureza y su germinación. Lo anterior está basado en los fracasos en el establecimiento de las praderas por el uso de semillas de mala calidad.

El factor calidad de la semilla debe tenerse en cuenta en las forrajeras, y, especialmente, en las leguminosas que presentan problemas en la germinación, debido a un alto porcentaje de semillas duras, el cual varía entre especies y aún entre muestras, dependiendo de factores genéticos, climáticos y de manejo.

Las semillas de Centrosema, como ocurre con las leguminosas forrajeras en general, presentan un tegumento o testa dura que las hace impermeables al agua. Esta ca-

1 Presentado en la XXXV Reunión Anual del PCCMCA, San Pedro Sula, Honduras, 1989.

2 Investigador Asociado, Unidad de Semillas, Centro Internacional de Agricultura Tropical (CIA1), Apartado aéreo 6713, Cali, Colombia.

${ }^{3} \mathrm{El}$ abstract es traducción del compendio. Elaborado por el Comité Editorial para mostrar el formato de presentación de los artículos.

Publicado en Agronomía Mesoamericana, Vol. 1 (1990) 
racterística es benéfica para la supervivencia de la especie en condiciones adversas de clima. Pero, por otro lado, en condiciones climáticas favorables, se constituye en una barrera más para una buena formación inicial de la pradera, puesto que reduce la germinación natural de las semillas. Por tanto, para aumentar el índice de germinación de las semillas de Centrosema es necesario escarificarlas.

Existen diferentes métodos para romper la dureza de la testa de estas semillas, ya sea mediante escarificación mecánica o química, usando un corte en la testa, o simplemente agua caliente (Aragao y Costa, 1983).

\section{OBJETIVOS}

Existe poca experiencia con especies como Vichada, C. brasilianum, y C. macrocarpum en cuanto a su calidad fisológica al haber sido sometidas a un tratamiento de escarificación química y luego almacenadas durante largo tiempo. Por esta razón, se decidió adelantar el presente estudio, con el fin de lograr los siguientes objetivos:

- Evaluar el efecto de la escarificación química con ácido sulfúrico sobre la germinación y emergencia en tres especies de Centrosema.

- Comparar el comportamiento de semillas con y sin escarificación química (con ácido sulfúrico), a través del tiempo de almacenamiento.

- Analizar la interacción de especies por tratamiento, a través del tiempo de almacenamiento.

\section{REVISION DE LITERATURA}

Las leguminosas son componentes importantes de las pasturas tropicales, pues aportan a la fertilidad del suelo, fijando nitrógeno del aire, el cual puede ser aprovechado por las gramíneas asociadas para aumentar su producción y calidad nutritiva (ICA, 1987).

La producción de semillas de leguminosas forrajeras tropicales varía entre especies. En la mayoría, la producción se presenta en crecimiento postrado; algunas, con crecimiento voluble necesitan soportes para producir una mayor cantidad de semillas. Esto ocurre con las especies de Centrosema, entre las cuales sobresalen $C$. acutifoliun, $C$. brasilianum y $C$. macrocarpum.

El cultivar Vichada fue colectado en la Comisaría del Vichada (Colombia). Se adapta desde el nivel del mar hasta $1,400 \mathrm{msnm}$, en regiones con precipitaciones de 1,000 a 2,500 mm. Se adapta a suelos ácidos de baja fertilidad natural. La producción de semillas varía entre 30 y $150 \mathrm{~kg} / \mathrm{ha}$, con una producción de forraje que oscila entre 900 y $2,000 \mathrm{~kg} / \mathrm{ha}$ de materia seca. El contenido de proteína alcanza un $25 \%$. Se puede asociar con el cultivar Carimagua, Braquiaria común, o Llanero (ICA, 1987).
C. brasilianum CIATNo. 5234 tiene un buen potencial como planta forrajera, tanto en regiones de trópico semiárido, como en regiones de suelos ácidos y de baja fertilidad (Clements et al. 1988). Este material tiene su origen en Bahía, Brasil y se adapta a zonas húmedas de la Amazonia, con una tolerancia aceptable a la sequía. Tiene una gran capacidad para producir semillas, con rendimientos entre 200 a $800 \mathrm{k} / \mathrm{ha}$. Es una especie de buen valor nutritivo, particularmente en cuanto a su contenido de proteína cruda (Belalcázar y Schultze-Kraft, 1986).

C. macrocarpum CIAT No. 5713 fue colectado en Anzoátegui, Venezuela en febrero de 1981. Es una planta perenne robusta, adaptada a suelos ultisoles ácidos (pH 4.1, 89\% de saturación de aluminio). Posee un alto valor nutritivo y un buen potencial para producir semillas (Schultze- Kraft et a1,1987).

En comparación con las gramíneas tropicales, las semillas de las leguminosas tropicales cosechadas son más limpias y tienen un alto poder germinativo. En consecuencia, los problemas de la calidad de las semillas no son tan serios con las leguminosas.

Sin embargo, la mayoría de las especies de leguminosas tropicales desarrollan una corteza dura en la semilla que reduce sustancialmente la germinación rápida y uniforme, si no se hace tratamiento para disminuir el efecto de la dureza (Purcelt, 1975). Este autor menciona que la mayoría de las especies de leguminosas contienen cerca del $80 \%$ de semilla dura y relaciona tres métodos principales de tratamiento para reducir la dureza: Tratamientos basados en métodos mecánicos, en métodos químicos, o en calor y remojo.

Puesto que no siempre es posible conocer la procedencia de la semilla ni si ésta ha sido cosechada mecánica o manualmente, como regla general se recomienda evaluar la germinación de la semilla. En el evento de que el resultado obtenido no sea superior al $40 \%$, es obvio que la semilla se beneficiará mucho con el tratamiento para reducir la dureza.

\section{Escarificación mecánica}

La escarificación mecánica se reduce a dañarla corteza de la semilla para permitir la entrada de humedad una vez que se haya sembrado. Cuando las cantidades de semillas son pocas, se puede raspar la semilla entre dos superficies a las cuales se les ha pegado papel de lija. La operación debe continuarse hasta que una pequeñísima cantidad de las sernillas se rompan o astillen. En el caso de semillas de tamaño grande, se puede partir o mellar una pequeña parte de la cubierta con un par de tijeras.

\section{Escarificación química}

La escarificación química comprende el uso de ácido sulfúrico concentrado; también se puede usar ácido 
sulfúrico negro comercial sin diluir. La semilla se mezcla con el ácido en un recipiente rodeado exteriormente con agua y se agita continuamente durante el tiempo requerido - 10 a 20 minutos según la especie. La semilla se agita continuamente en un baño de agua debido a que el proceso libera mucho calor. En otra forma, el calor liberado sería suficiente para deteriorar el embrión de la semilla. Aunque se pueden utilizar otros metales, se recomienda usar un recipiente de cobre para el tratamiento con ácido, es recomendable porque este metal $\mathrm{u}$ un buen conductor de calor y lo difunde rápidamente hacia el exterior. Después del tratamiento se debe lavar bien con agua todo residuo de ácido en las semillas, con el objeto de evitar menoscabo al Rhizobium inoculado posteriormente.

\section{Escarificación por calor y remojo}

Un método simple, utilizado para reducir la dureza de la corteza de las semillas de la mayoría de las especies de leguminosas consisten en embeber la semilla en agua durante 24 horas, secarla luego rápidamente y proceder a la siembra inmediatamente. Sin embargo, una deficiencia de este sistema es la urgencia en sembrar tan pronto se hace el tratamiento.

En general, las semillas de leguminosas tropicales que han recibido tratamiento para reducir el factor dureza y que han permanecido en reposo durante un mínimo de 3 meses después de cosechadas, deberán exhibir entre el 50 y $80 \%$ de germinación, con excepción de las especies de Stylosanthes, las cuales presentan un $40 \%$ en promedio en las condiciones mencionadas.

Trabajos realizados con $C$. pubescens, en los que las semillas fueron escarificadas con ácido y mecánicamente, encontraron que solamente el $30 \%$ de las semillas examinadas germinaron en condiciones favorables de humedad y temperatura. El porcentaje de germinación aumentó en más del $80 \%$ después de los tratamientos (Pe et al, 1975).

La viabilidad de semillas de $C$. pubescens almacenadas a $33^{\circ} \mathrm{C}$ y $90 \%$ de humedad relativa se deterioró rápidamente, corno resultado de una mayor actividad de hongos durante el almacenamiento. El mismo autor sometió semillas a escarificación química y las guardó a $20^{\circ} \mathrm{C}$ y $40 \%$ de humedad relativa, encontrando que su viabilidad se mantuvo hasta los 6 meses. Encontró, además, que la disminución en b viabilidad dependió de las condiciones de la semilla antes del almacenamiento y no durante el mismo.

Varias especies tropicales (entre ellas $C$. pubescens fueron sometidas a tratamientos de a) Escarificación química con ácido sulfúrico, b) Agua caliente $\left(80^{\circ} \mathrm{C}\right)$ durante 20 minutos, y e) Testigo, sin tratamiento. Se encontró que tanto la escarificación química como el tratamiento con agua caliente favorecieron el incremento en la germinación y el vigor, en comparación con semillas sin tratar, almacenadas a $8^{\circ} \mathrm{C}$ (Cabrales y Bernal, 1983).
Almeida et al, (1979) trabajaron con cinco especies de leguminosas (Glycine wightii, Macroptilium atropurpureum, Calopogonium muconoides, Centrosema pubescens y Pueraria phaseoloides) utilizando varios métodos de escarificación con ácido sulfúrico concentrado, calor, papel lija y agua caliente, y encontraron que todas las especies presentaban un alto porcentaje de semillas duras y que con excepción de C. pubescens, éstas no aumentaron su germinación durante, el almacenamiento. La diferencia de color en los lotes de semillas se relacionó con la existencia de semillas muertas.

Seiffert (1982) estableció que semillas de especies de Cajanus, Calopogonium, Centrosema, Leucaena, Nenotonia, Pueraria y Stylosanthes con una germinación inicial del $60 \%$, deben ser escarificadas con otros métodos (ácido sulfúrico, comercial, soda cáustica comercial al $20 \%$, o agua a $100^{\circ} \mathrm{C}$ ) para estimular la germinación. Las pruebas posteriores de deben dar resultadoss entre el 80 a $90 \%$.

Semillas de C. brasilianum CIAT No. 5234 escarificadas con ácido sulfúrico comercial presentaron una germinación del $80 \%$, comparado con un $14 \%$ de la semilla sin escarificar. Estos valores se encontraron después de cosechada la semilla. Semilla sin escarificar de esta misma especie, almacenada durante 16 meses, exhibió una germinación del 33\% (Búrbano y Giraldo, 1988).

\section{MATERIALES}

Las semillas utilizadas para realizar el tratamiento de escarificación química con ácido sulfúrico fueron cosechadas en la región de Valledupar, departamento del Cesar (Colombia) a una latitud de $10^{\circ} 20^{\prime} \mathrm{N}$, en febrero de 1987.

Las especies utilizadas fueron: $C$. acutifolium CIAT No. 5277 cv. Vichada, C. brasilianum CIAT No. 5234 y C. macrocarpum CIAT No. 5713. El trabajo incluyó los siguientes tratamientos:

a. Semilla no escarificada y almacenada al ambiente.

b. Semilla no escarificada y almacenada en condiciones de cuarto frío.

c. Semilla escarificada y almacenada al ambiente.

d. Semilla escarificada y almacenada en cuarto frío.

e. Semilla almacenada al ambiente sin escarificar y luego escarificada cada mes.

f. Semilla almacenada en cuarto frío sin escarificar y luego escarificada cada mes.

Se utilizó un diseño experimenta] completamente aleatorio y un análisis de covarianza para conocer el comportamiento mensual de los diferentes tratamientos y especies a través del tiempo de almacenamiento.

\section{METODOLOGÍA UTILIZADA}

Se utilizó semilla de las tres especies, clasificada 
con un $99 \%$ de pureza física y un $95 \%$ de viabilidad en tetrazolio. Se secó la semilla al sol hasta que alcanzara un contenido de humedad del $12 \%$. Luego se procedió a escarificar las semillas, de acuerdo con los tratamientos establecidos, de la siguiente manera: Se colocaba la semilla en un recipiente plástico y se humedecía con ácido sulfúrico, calculando unos $100 \mathrm{cc} / \mathrm{kg}$ de semilla. Se observó que no es la cantidad, sino el tiempo de escarificación el que determina un buen efecto en términos de disminución del número de semillas duras. Se escarificaron durante 15 minutos, revolviendo la masa de semillas continuamente. Luego se lavaron las semillas con agua en abundancia para evitar quemarlas y eliminar cualquier residuo de ácido que pudiera afectar la germinación. Finalmente, se seco la semilla al sol durante 2 a 3 horas, asegurando una humedad del $12 \%$.

La semilla almacenada al ambiente fue colocada en el laboratorio. Aquella almacenada en cuarto frío tenía unas condiciones de 18 a $20^{\circ} \mathrm{C}$ y un 50 a $60 \%$ de humedad relativa. Se almacenó en bolsas de polipropileno durante 19 meses.

Cada mes se evaluó la calidad fisiológica de la semilla, mediante pruebas de germinación, utilizando cuatro repeticiones de 50 semillas y papel tipo Anchor como sustrato. Se colocaron a germinar en una cabina con temperaturas alternas $\left(20\right.$ y $\left.30^{\circ} \mathrm{C}\right)$, realizando conteos a los 4 y 9 días, En el último conteo se estableció el número de semillas duras, frescas y muertas, además del número de plántulas normales y anormales, producto del primer y segundo conteo.

Se estableció la prueba de emergencia a los 19 meses para todos los tratamientos, para comparar con los resultados de germinación bajo condiciones controladas.

\section{RESULTADOS Y CONCLUSIONES}

Realizando el análisis de varianza, se encontró que los resultados fueron altamente significativos $(\mathrm{P}<0.001)$ para todos los tratamientos, especies e interacciones durante los 19 meses de almacenamiento, tanto para las plántulas normales, como para las semillas duras, al igual que los otros componentes de la prueba de germinación. La prueba de Duncan, demostró que $C$. acutifolium 5277 se comportó diferente a las otras dos especies en términos de plántulas normales $(63 \%, \mathrm{P}<0.05)$ en todos los tratamientos. En cuanto a semillas duras, C. macrocarpum presentó el mayor número de semillas duras $(19 \%$, Duncan $\mathrm{P}<0.05)$ a través de todos los meses y tratamientos.

Analizando los tratamientos mediante la prueba de Duncan $(\mathrm{P}<0.05)$ se encontró que el tratamiento de semilla guardada en cuarto frío y escarificada fue el mejoren términos de plántulas normales $(80 \%)$ en relación al tratamiento testigo almacenado al ambiente, el cual presentó un $46 \%$ de plántulas normales. Todos los tratamientos fueron diferentes $(\mathrm{P}<0.05)$ en términos de se- millas duras, encontrando que el tratamiento de semilla almacenada al ambiente alcanzó un valor más alto de dureza, en comparación con los demás tratamientos $(372 \%)$ a través del tiempo $(\mathrm{P}<0.05)$.

En el Cuadro 1 se presenta el comportamiento general de las tres especies, a través de los 19 meses, en términos del porcentaje de plántulas normales y de semillas duras. A pesar de la poca diferencia, se encontraron diferencias entre tratamientos, según la prueba de Duncan $(\mathrm{P}<0.05)$.

Cuadro 1. Porcentaje de plántulas normales y semillas duras de las tres especies a través de los 19 meses y los seis tratamientos.

\begin{tabular}{|c|c|c|c|}
\hline $\begin{array}{c}\text { Plántulas } \\
\text { Normales } \\
\quad(\%)\end{array}$ & Meses & $\begin{array}{c}\text { Semillas } \\
\text { Duras } \\
(\%)\end{array}$ & Meses \\
\hline 701 & 10 & 20 & 1 \\
\hline 69 & 11 & 19 & 2 \\
\hline 68 & 9 & 19 & 3 \\
\hline 68 & 12 & 19 & 5 \\
\hline 68 & 13 & 18 & 4 \\
\hline 67 & 8 & 18 & 6 \\
\hline 67 & 7 & 15 & 7 \\
\hline 66 & 2 & 15 & 8 \\
\hline 65 & 3 & 14 & 9 \\
\hline 65 & 15 & 13 & 10 \\
\hline 65 & 17 & 13 & 11 \\
\hline 65 & 14 & 12 & 15 \\
\hline 65 & 18 & 12 & 14 \\
\hline 64 & 16 & 12 & 16 \\
\hline 64 & 1 & 11 & 17 \\
\hline 63 & 6 & 10 & 12 \\
\hline 62 & 5 & 10 & 13 \\
\hline 61 & 4 & 9 & 18 \\
\hline 61 & 19 & 8 & 19 \\
\hline
\end{tabular}

Promedios; unidos por la misma barra no difieren significativamente según Duncan $(\mathrm{P}<0.05)$.

Se realizó el análisis de covarianza con el fin de predecir la tendencia de cada una de las especies sometidas a los diferentes tratamientos a través de los 19 meses y no se observó aumento en el porcentaje de plántulas normales con relación al inicio del ensayo en el Tratamiento A. Se destaca C. macrocarpum con un incremento $(35$ al $54 \%)$ más representativo $(\mathrm{P}=0.0001)$ que el de C. acutifolium (45a150\%,P. $=0.054)$ y $C$. brasilianum (42 al 55\%, $\mathrm{P}=0.02$ ). En el 1 Tratamiento $\mathrm{B}$, se observó un efecto significativo en términos de germinación para las tres especies: C. macrocarpum, (del 30 al $68 \%, \mathrm{P}=0.000$ 1); y C. brasilianum y C. acutifolium, un incremento mínimo porcentual, pero también significativo $(\mathrm{P}=0.0001)$. En el Tratamiento $\mathrm{C}$ se observó que hubo un efecto significativo $(\mathrm{P}=0.0001)$ para las tres especies, destacándose la reducción de plántulas normales de C. brasilianum (del 80 al 60\%) y de C. aculifolium (del 80al 40\%, con una caída del $70 \%$ a sólo 5 meses de almacenamiento). En el Tratamiento D, sólo se observó una disminución en plántulas normales 
en el caso de $C$. acutifolium $(\mathrm{P}=0.000$ 1), Los resultados en el Tratamiento $\mathrm{E}$ fueron significativos $(\mathrm{P}=$ 0.0001), presentándose una disminución del 90 al $70 \%$ de plántulas normales en $C$. brasilianum y $C$. acutifolium. Los resultados del Tratamiento $\mathrm{F}$ fueron significativos $(\mathrm{P}=0.0001)$ para $C$. brasilianum y $C$. macrocarpum, con un aumento en el porcentaje de plántulas normales a través de los 19 meses. Lo anterior indica que las temperaturas bajas mantienen la dureza, lo cual ofrece ventajas en el almacenamiento a largo plazo.

Los resultados anteriores indican que la escarificación química con ácido sulfúrico es benéfica. Se debe observar el momento apropiado para hacer la escarificación y el tiempo que ésta debe durar. La presencia de plántulas anormales a partir del mes 9 en la mayoría de los tratamientos, sugiere que a medida que el lote envejece, se debe disminuir el tiempo de escarificación. En el Cuadro 2 se presentan los resultados de germinación y emergencia, a los 19 meses de almacenamiento. Mediante el análisis de varianza, se encontró que los resultados fueron altamente significativos $(\mathrm{P}<0.001)$. Se hizo la Prueba de Duncan para determinar la diferencia entre tratamientos; se pudo observar que el Tratamiento $\mathrm{C}$ fue el menos conveniente para mantener la calidad de la semilla de las especies evaluadas. El Tratamiento F resultó ser el mejor para las tres especies, rompiendo la dureza entre 40 y $80 \%$, según la especie. Se pudo observar que las plántulas normales evaluadas a los 19 meses no presentaron el mismo vigor de las plántulas evaluadas cuando se inició el ensayo.

Se puede concluir que la semilla sin escarificar químicamente, difícilmente disminuye su dureza naturalmente. Las condiciones controladas de almacenamiento mantienen la calidad de la semilla y facilitan el rompimiento de la dureza mediante un menor tiempo de escarificación. No se debe guardar semilla escarificada en condiciones ambientales no controladas, pues

Cuadro 2. Porcentajes* de germinación (G). y emergencia (E) de las tres especies de Centrosema evaluadas después de 19 meses de almacenamiento, según los tratamientos aplicados

\begin{tabular}{ccccccc}
\hline Trat.** & \multicolumn{2}{c}{ C. brasilianum } & \multicolumn{2}{c}{ C. acutifoliun } & \multicolumn{2}{c}{ C. macrocarpun } \\
& CIAT 5234 & \multicolumn{2}{c}{ CIAT 5277 } & \multicolumn{2}{c}{ CIAT 5713 } \\
& G & $\mathbf{E}$ & $\mathbf{G}$ & $\mathbf{E}$ & $\mathbf{G}$ & $\mathbf{E}$ \\
\hline $\mathrm{A}$ & $54 \mathrm{c}$ & $42 \mathrm{~b}$ & $39 \mathrm{~d}$ & $30 \mathrm{bc}$ & $52 \mathrm{c}$ & $34 \mathrm{~d}$ \\
$\mathrm{~B}$ & $55 \mathrm{c}$ & $48 \mathrm{~b}$ & $49 \mathrm{c}$ & $34 \mathrm{~b}$ & $59 \mathrm{bc}$ & $58 \mathrm{c}$ \\
$\mathrm{C}$ & $45 \mathrm{~d}$ & $28 \mathrm{c}$ & $27 \mathrm{c}$ & $6 \mathrm{~d}$ & $61 \mathrm{~b}$ & $56 \mathrm{c}$ \\
$\mathrm{D}$ & $88 \mathrm{a}$ & $72 \mathrm{a}$ & $54 \mathrm{~b}$ & $38 \mathrm{~b}$ & $78 \mathrm{a}$ & $72 \mathrm{~b}$ \\
$\mathrm{E}$ & $64 \mathrm{~b}$ & $66 \mathrm{a}$ & $58 \mathrm{~b}$ & $18 \mathrm{c}$ & $75 \mathrm{a}$ & $66 \mathrm{bc}$ \\
F & $85 \mathrm{a}$ & $76 \mathrm{a}$ & $74 \mathrm{a}$ & $62 \mathrm{a}$ & $78 \mathrm{a}$ & $86 \mathrm{a}$ \\
\hline
\end{tabular}

* Los valores seguidos por la misma letra no difieren significativamente (Prueba de Duncan, $\mathrm{P}<0.05$ ).

** A = Semilla no escarificada y almacenada al ambiente; $\mathrm{B}=$ Semilla no escarificada y almacenada en cuarto frío; $\mathrm{C}=$ Semilla escarificada y almacenada al ambiente; $\mathrm{D}=$ Semilla escarificada y almacenada en cuarto frío; $\mathrm{E}=$ Semilla almacenada al ambiente inicialmente sin escarificar, y luego escarificada cada mes; y F = Semilla almacenada en cuarto frío inicialmente sin escarificar, y luego escarificada cada mes. la disminución en calidad (germinación) se inicia a los 5 meses. La especie $C$. acutifolium fue la que presentó una mayor facilidad para romper la dureza, lo que podría representar una desventaja, puesto que inicia más rápidamente su deterioro y pérdida de calidad.

Se recomienda, entonces, no almacenar al ambiente la semilla escarificada, ya que el número de semillas muertas empieza a incrementarse. Siempre que se vaya a realizar una siembra de estos materiales, es necesario escarificarlos; de lo contrario, se debe ajustar la tasa de siembra, puesto que ellos no rompen la dureza por medios naturales. Solamente $C$. macrocarpum almacenada en cuarto frío mostró una buena respuesta al tratamiento de escarificación. Si se pretende escarificar unos días antes de la siembra, es necesario tener buenas condiciones de almacenamiento para la semilla.

\section{BIBLIOGRAFIA}

ALMEIDA, LDA. DE, MAEDA, J.A.; FALIVENE, S.M.P. 1979. Efeitoe de métodos de escarificao na germinacao de semenetes de cinco leguminosas forrageiras. Bragantia 38(9):83-96. Port., Res. Port, Ingl., 14 Refs.

ARAGAO, W.M.; COSTA, B.M., DA. 1983. Evaluación de métodos de escarificación en la germinación de semillas de Centrosema pubescens. Aracaju-SE, Brasil, Empresa Brasileira de Pesquisa Agropecuaria. Camunicado Técnico No. 6, 3 p. Port.

BELALCAZAR, J.; SCHULUE-KRAFT, R. 1986. Centrosema brailianum (L.) Benth: descripción de la especie y evaluación agronómica de siete ecotipos. In Pasturas Tropicales, Boletín, Vol. 8, No. 3.

BURBANO, E.A.; GIRALDO, G. 1988. Sistema de soporte, densidad de siembra y fecha de cosecha de semillas de Centrosema brasilianum In Pasturas Tropicales, Vol. 10, No. 2.

CABRALES, R.; BERNAL, J. 1983. Effect of different systems of seed treatment, packing, and storage on vigor and germination of five tropical forage legumes. In Smith, J.A.; Hays, V.W. (Eds.), International Grassland Congress, 14th., Lexington, Kentucky, 1981. Proceedings, Boulder, Colorado, Westview Press. p. 263 -265. Ingl.

CLEMEN7S, RJ; W7LLIAMS, R.J.; GROF, B; HACKER, I.B. 1983. Centrosema. In Burt, R.L.; Rotar, P.P.; Walker, J.L.; Silvey, M. W. (Eds). The role of Centrosema, Desmodium, and Stylosanthes in improving tropical pasture. Boulder, Colorado, Westview Press, Westview Tropical Agriculture Series, No. 6, p. 69-96.

INSTITUTO COLOMBIANO AGROPECUARIO (ICA). 1987. Centrosema. Vichada. Boletín Técnico No. 152, 13 p.

PE, W.; HILL, MJ.; JOHNSTON, M.E.H. 1975. Efecto del almacenamiento y tratamiento de la semilla en la germinación de semillas de Centrosema pubescens. New Zealand Journal of Experimental Agriculture 3(1):85-89. Ing., 16 Refs. Ilus.

PURCELL, D. 1975. Producción, almacenamiento y tratamiento de semillas de forrajeras. In Seminario sobre producción de semillas de forrajeras. Bogotá, Colombia. Trabajos. Bogotá, Instituto Interamericano de Ciencias Agrícolas de la OEA. Serie Informes de Reuniones, Cursos y Conferencias No. 79, 1975. p. 61-88. Esp. 13 Refs. Ilus.

SEIFFERT, N.F. 1982- Métodos para la escarificación de semillas de leguminosas forrajeras tropicales. Campo Grande-MS, Brasil, Empresa Brasileira de Pesquisa Agropecuaria. Centro Nacional de Pesquisa de Gado de Corte. Comunicado Técnico No. 13. 6 p. Port.

SCHULTZE-FRAKT, R.; ARENAS, J.A.; FRANCO, M.A.; BELALCAZAR, J.; ORTIZ, J. 1987. Catálogo de germoplasma de especies forrajeras tropicales. 4a. ed., CIAT, Cali, Colombia. Tomos 1-III, 1436p. 